\title{
BMJ Open Does contemporary exercise-based cardiac rehabilitation improve quality of life for people with coronary artery disease? A systematic review and meta- analysis
}

\author{
Gordon McGregor (DD , ${ }^{1,2,3}$ Richard Powell, ${ }^{1,3}$ Peter Kimani, ${ }^{1,2}$ Martin Underwood ${ }^{1,2}$
}

To cite: McGregor G,

Powell R, Kimani P, et al. Does contemporary exercise-based cardiac rehabilitation improve quality of life for people with coronary artery disease? A systematic review and meta-analysis. BMJ Open 2020;10:e036089. doi:10.1136/ bmjopen-2019-036089

- Prepublication history and additional material for this paper are available online. To view these files, please visit the journal online (http://dx.doi. org/10.1136/bmjopen-2019036089).

Received 29 November 2019 Revised 02 March 2020 Accepted 01 May 2020

Check for updates

(c) Author(s) (or their employer(s)) 2020. Re-use permitted under CC BY-NC. No commercial re-use. See rights and permissions. Published by BMJ.

${ }^{1}$ University Hospitals Coventry $\&$ Warwickshire NHS Trust, Coventry, UK

${ }^{2}$ Warwick Medical School, University of Warwick, Coventry, UK

${ }^{3}$ Centre for Sport, Exercise \& Life Sciences, Coventry University, Coventry, UK

Correspondence to

Dr Gordon McGregor;

gordon.mcgregor@uhcw.nhs.uk

\section{ABSTRACT}

Objectives To determine the effect of contemporary exercise-based cardiac rehabilitation on generic and disease-specific health related quality of life for people with coronary artery disease.

Design Systematic review and meta-analysis.

Study eligibility criteria Randomised controlled trials testing exercise-based cardiac rehabilitation versus no exercise control that recruited after 31 December 1999. On 30 July 2019, we searched the Cochrane Central Register of Controlled Trials, MEDLINE (Ovid), Embase (Ovid) and CINAHL (EBSCO) databases.

Study appraisal and synthesis Studies were screened for inclusion by two independent reviewers. Risk of bias was assessed using the Cochrane risk of bias tool. Data were reported as pooled means $(95 \% \mathrm{Cl}$ for betweengroup difference.

Results We identified 24 studies $(n=4890)$. We performed meta-analyses for 15 short-term and 9 medium-term outcomes (36-Item Short Form Survey Instrument (SF36), EuroQol-5D (EQ-5D) and MacNew, a cardiac-specific outcome). Six short-term and five medium-term SF-36 domains statistically favoured exercise-based cardiac rehabilitation. Only for two short-term SF-36 outcomes, 'physical function' (mean difference 12.0, 95\% Cl 4.4 to 19.6) and 'role physical' (mean difference 16.9, 95\% Cl 2.4 to 31.3), did the benefit appear to be clinically important. Meta-analyses of the short-term SF-36 physical and mental component scores, EQ-5D and MacNew and the medium-term SF-36 physical component score, did not show statistically significant benefits. Only two studies had a low risk of bias ( $n=463$ participants).

Conclusions and implications of key findings There is some evidence of a short-term benefit of contemporary exercise-based cardiac rehabilitation on quality of life for people with coronary artery disease. However, the contemporary data presented in this review are insufficient to support its routine use.

\section{INTRODUCTION}

Coronary artery disease (CAD) is the leading cause of death worldwide. ${ }^{1}$ Over the past 30 years, advances in interventional and secondary preventative cardiology have
Strengths and limitations of this study

- To our knowledge, this is the most comprehensive systematic review and meta-analysis of contemporary exercise-based cardiac rehabilitation for people with all manifestations of coronary artery disease.

- We conducted meta-analyses for 15 short-term and 9 medium-term outcomes.

- We assessed risk of bias for all included studies using the Cochrane risk of bias tool.

- Data had a high level of statistical heterogeneity and the majority of studies were identified as having 'some concerns' or 'high risk' in relation to the risk of bias assessment.

- Data were insufficient to analyse at distinct timepoints, thus were pooled as short-term (1-6 months) or medium term (8-12 months).

dramatically improved survival for people with CAD. ${ }^{23}$ In high-income countries, living with $\mathrm{CAD}$, as a long-term condition, is now common. Of the 200000 people who have a myocardial infarction annually in the UK, 7 out of 10 survive. In 2018, there were over 900000 survivors of myocardial infarction and 2.3 million people living with $\mathrm{CAD}$ in the $\mathrm{UK}^{4}$ This longevity after myocardial infarction represents a substantial and increasing burden on healthcare resource. There is a need for medical and lifestyle interventions that improve quality of life (QoL), maintain physical and psychosocial independence, and reduce long-term health and social care utilisation.

Cardiac rehabilitation (CR) has long been considered integral to the management of CAD. ${ }^{5}$ Exercise training in conjunction with cardiovascular risk factor management, psychosocial support and behaviour change ('comprehensive' CR) are the core components of a complex health and lifestyle 
intervention, which is unreservedly advocated in international guidelines and policy. ${ }^{67}$ Multiple meta-analyses incorporating trials spanning 1975-2018 reported favourable effects on functional capacity, hospital readmissions and mortality. ${ }^{8-12}$

Nevertheless, our 2018 systematic review (22 randomised controlled trials (RCTs), N=4834), which only included RCTs of 'contemporary' exercise-based CR that recruited after the end of 1999, found that the CR programmes tested had no effect on all-cause mortality (risk difference $0.0,95 \%$ CI -0.02 to 0.01 ), and only a small effect on hospital readmissions of borderline statistical significance. ${ }^{13}$ A 2018 network meta-analysis, while showing a reduction in mortality when including studies from 1975 to present day, found a non-significant reduction in mortality for studies published after 2001. ${ }^{12}$ Existing data do not support the continued delivery of exercise-based CR in its current form where the intention is to reduce mortality or prevent hospital readmissions in CAD. For the continued use of these programmes to be justified for people with CAD, a paradigm shift in their stated aims is required.

In an ageing, multimorbid population, QoL, defined by $\mathrm{WHO}$ as 'an individual's perception of their position in life in the context of the culture and value systems in which they live and in relation to their goals, expectations, standards and concerns', ${ }^{14}$ is a key priority for patients and healthcare providers. Patient-reported outcomes such as QoL are unique in providing the patient's perspective on the efficacy of medical or lifestyle interventions. ${ }^{15}$ Furthermore, any change is tangible and subjective, thus patients can themselves perceive and report any benefit associated with CR. Therefore, CR should perhaps be judged on its ability to add 'life to years' rather than 'years to life'. Nearly all previous systematic reviews have considered QoL data for exercise-based CR to be insufficient or unsuitable for meta-analysis due to considerable heterogeneity in outcome measures and reporting. A 2016 Cochrane review ${ }^{9}$ concluded that present data demonstrated improvement in at least one QoL domain in $65 \%$ of studies, and improvement in half or more of the reported domains in $25 \%$ of studies.

A 2018 meta-analysis (41 RCTs, N=11 747), pooling a range of measures and CR interventions from studies between 1975 and 2017, found that exercise training was associated with a small positive effect on QoL, but, overall, 'psychosocial management' was more effective. ${ }^{16}$ A 2018 Cochrane review of exercise-based CR for angina pectoris was unable to draw conclusions on the impact of this intervention on QoL. ${ }^{17}$ Subsequently, a 2019 metaanalysis reported exercise to be effective in reducing anxiety and depression following myocardial infarction. ${ }^{18}$ However, in a review of prospective cohort studies, ${ }^{19}$ people with depression were four times less likely to participate in CR and seven times more likely to drop out. A 2019 systematic review (14 RCTs, N=1739) of CR for people following acute coronary syndrome, published when this paper was being prepared for submission, included eight studies in a meta-analysis and concluded that there were clinically important positive effects on two 36-Item Short Form Survey Instrument (SF-36) domains at 6 months (role physical and general health) and one domain at 12 months (physical function). ${ }^{20}$

The objective of this systematic review and metaanalysis was to determine the effect of exercise-based CR on health-related QoL in all people with CAD, in the era of modern medical management.

\section{METHODS}

The methodology for our systematic review and metaanalysis adhered to the Preferred Reporting Items for Systematic Reviews and Meta-Analysis guidelines and the study was registered on PROSPERO (CRD42018110197).

\section{Search strategy and methodology}

First, we reviewed all studies included in the most recent Cochrane systematic review of exercise-based CR in coronary heart disease. ${ }^{9}$ Second, we assessed the 93 studies listed as 'excluded' in the Cochrane review to identify any additional studies that met our inclusion criteria. Third, on 30 July 2019, we searched the Cochrane Central Register of Controlled Trials, MEDLINE (Ovid), Embase (Ovid) and CINAHL (EBSCO) databases using the strategy used in the Cochrane Systematic Review (online supplementary appendix 1 ).

Results from our three predefined sources were individually examined to determine inclusion or exclusion. We retrieved abstracts, full-text manuscripts and supplementary material where necessary, and hand searched reference lists of the subsequently included articles (and other recent systematic reviews) to identify additional studies of interest. Two reviewers (GMcG and RP) independently undertook screening of the resultant citations, abstracts and manuscripts, with disputes mediated by a third reviewer (MU). Where data were missing or inappropriately presented, we requested additional information from lead and corresponding authors by email, on multiple occasions.

\section{Study inclusion criteria}

Our overall aim was to identify RCTs testing an exercisebased CR intervention against non-exercise usual care, with QoL as an outcome measure, which recruited after 31 December 1999. The rationale for excluding studies recruiting prior to the year 2000 is detailed elsewhere. ${ }^{13}$ Briefly, we defined contemporary CR as postdating the widespread adoption of primary percutaneous coronary intervention and the 'modern' pharmacology outlined in the Joint British Society recommendations for the Prevention of Coronary Heart Disease in Clinical Practice. ${ }^{21}$ Including studies that recruited after the end of 1999 allowed sufficient time for these innovations to become commonplace.

\section{Design}

We identified RCTs testing an exercise-based CR intervention against non-exercise usual care, which reported 
outcomes at any time-point following completion of the intervention. Previous reviews assessing mortality and hospitalisation have only included studies with at least 6 months follow-up. ${ }^{9}$ For QoL indicators, we considered outcome measures at any time-point following completion of CR to be of interest. We excluded abstracts, conference proceedings, theses and non-English language publications.

\section{Participants}

We included all studies where participants either had CAD confirmed with coronary angiography, had a diagnosis of angina pectoris, had undergone coronary revascularisation via either percutaneous coronary intervention or coronary artery bypass grafting, or had sustained a myocardial infarction.

\section{Interventions}

We defined interventions as exercise-based CR undertaken with or without supervision as a hospital inpatient, as a hospital outpatient, in a community venue or at home. Furthermore, the exercise programme could have been delivered in isolation or in combination with other educational, behavioural or psychosocial components constituting a 'comprehensive' multicomponent CR programme. We defined usual care as any intervention delivered to people with $\mathrm{CAD}$ that did not include a structured exercise component, that is, disease-specific education, smoking cessation, dietary advice or psychosocial support, delivered without supervised exercise training. We excluded studies in which both groups had completed a CR exercise training intervention prior to randomisation to an exercise intervention or a non-exercise control.

\section{Outcome measures}

Data were extracted from studies reporting betweengroup difference in QoL, collected with a generic or cardiovascular disease-specific, validated measure, for example, the SF-36 at any time-point post-CR. Measures were considered to be validated if there was evidence in the peer-reviewed literature that the instrument had been psychometrically tested for reliability, validity and/ or sensitivity.

\section{Data extraction and statistical analysis}

Any QoL data available at baseline and follow-up were extracted from studies that met the inclusion criteria. For each of the exercise-based CR and non-exercise usual care arms, the data extracted at each visit were the mean QoL score and the SD of the QoL score. If the means and SDs were not explicitly reported, they were extracted from line graphs (where possible) or derived from CIs. The SDs were computed assuming the CIs were obtained taking the point estimates to be normally distributed.

To pool the results from all studies for each QoL measure at a particular time-point, we fitted a randomeffects meta-analysis model in the $\mathrm{R}$ statistical program. ${ }^{22}$ The pooled results were summarised in forest plots. Where means and SDs in each arm were available for all included studies, the 'meta' package, with a command that requires specifying the mean and SD for each arm, was used to perform the meta-analysis. ${ }^{23}$ For the shortterm SF-36 Mental Health Component and Physical Health Component scores, two studies ${ }^{24}{ }^{25}$ reported the mean difference and the SE, thus it was not possible to extract the means and SDs. Therefore, the 'metafor' package, with a command that requires specifying mean difference and SE for each study, was used to perform the meta-analysis. ${ }^{26}$ This was the same approach to extracting EuroQol-5D (EQ-5D) data in another study. ${ }^{27}$ In these cases, if a study reported means and SDs, these were used to calculate the mean difference and the SE.

There were two options for defining the outcomes to be used to compare usual care and exercise-based CR at any particular time-point: (1) taking the outcome as the QoL score at each time-point or (2) taking the outcome as the change in QoL score from baseline. Some studies did not report baseline values or changes, and so the former definition was chosen. This enabled the inclusion of more studies in the meta-analyses.

\section{Assessment of risk of bias}

We performed a risk of bias assessment for all studies included in our meta-analyses using V.2 of the Cochrane risk of bias tool for randomised trials. ${ }^{28}$ Accordingly, risk of bias was assessed for general trial procedures and specifically for the QoL outcome of interest. Each trial was assessed against five domains of bias: (1) bias arising from the randomisation process; (2) bias due to deviations from intended interventions; (3) bias due to missing outcome data; (4) bias in measurement of the outcome and (5) bias in selection of the reported result. As per the Cochrane Handbook, ${ }^{28}$ an overall risk of bias score of 'low', 'some concerns' or 'high' was determined for each trial. 'Low' risk of bias was implied when all domains were scored 'low'. 'Some concerns' was implied when at least one domain was scored as 'some concerns'. 'High' risk of bias was implied when at least one domain was scored as 'high', or multiple domains were scored 'some concerns'. All studies were assessed independently by two reviewers (GMcG and RP) with discrepancies resolved by a third (MU).

\section{Patient and public involvement}

There was no patient and public involvement in this systematic review.

\section{RESULTS}

\section{Studies retrieved}

Thirteen studies in the Cochrane review ${ }^{24}$ 27 29-39 met our criteria and one study was identified from the Cochrane excluded studies list. ${ }^{40}$ Of 32 studies retrieved for full evaluation from our updated literature search, 3 were excluded as they were not RCTs, 14 because they did not use QoL as an outcome measure, 3 because participants completed structured CR prior to being randomised to a 


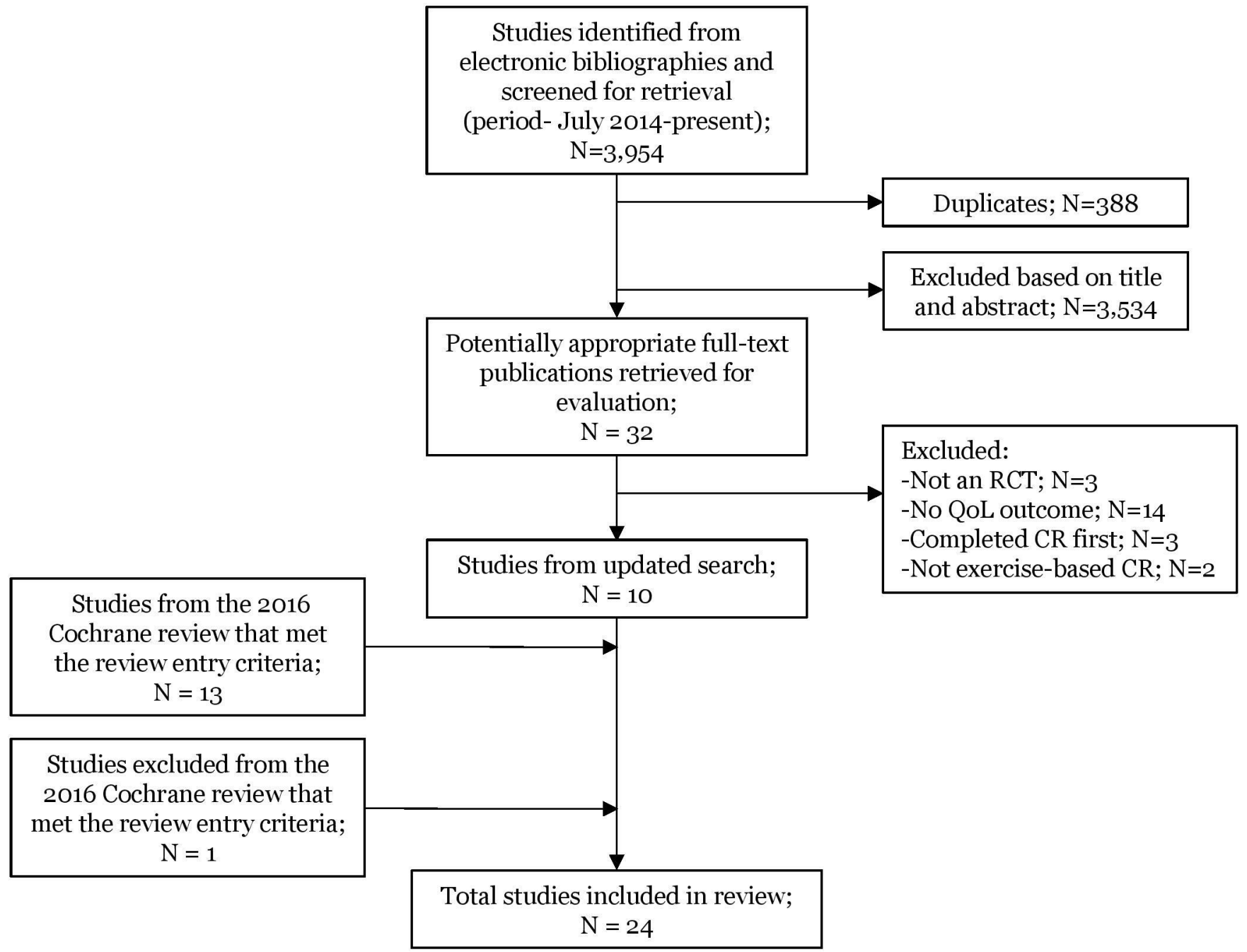

Figure 1 Preferred Reporting Items for Systematic Reviews and Meta-Analyses (PRISMA) diagram. CR, cardiac rehabilitation; QoL, quality of life; RCT, randomised controlled trial.

CR intervention or control group and 2 because they did not qualify as 'exercise-based CR': this left 10 studies. ${ }^{2541-49}$ A total of 24 studies $(\mathrm{N}=4,890)$ were suitable for inclusion (figure 1).

All studies reported QoL using at least one validated measure, and seven studies used two measures. Six different generic measures were used: SF-36 (14 studies), 12-item Short Form Survey (SF-12, 2 studies), EQ-5D (2 studies) and 20-item Short Form Survey (SF-20), 15 D Questionnaire, Time Trade Off Questionnaire 1 study each. Six cardiac-specific measures were used: MacNew QoL Questionnaire (four studies), HeartQoL Questionnaire (two studies) and Duke Activity Status Index (DASI), the Seattle Angina Questionnaire, Quality of Life Index-cardiac version III and Myocardial Infarction Dimensional Assessment Scale all one study each.

Data were reported at varying time-points and presented in numerous statistical formats, thus reducing the number of point estimates we could reliably include in each analysis. We contacted authors from eight studies 2427303337404244 to request provision of data in a format consistent with our meta-analysis protocol. A response was received from two authors stating that the data were not available. ${ }^{37} 42$

We performed meta-analyses at two time-points: shortterm (immediately postintervention, or as soon as possible thereafter, up to 6 months (1-6 months) ) and mediumterm (8-12 months postrandomisation). This allowed us to assess both the immediate postintervention effect of exercise-based CR, and the long-term effect. Where data were reported twice within the short-term timescale (ie, 3 and 6 months), ${ }^{35}$ data recorded closest to the end of the intervention period were included in the meta-analysis. We pooled data from studies using SF-36 and SF-12, henceforth SF-36. Data were sufficient to undertake metaanalysis for three measures: the SF-36 (eight domains and physical component score for the short-term and mediumterm time-points, plus the physical component score for the short-term time-point only), the EQ-5D (short-term only) and the MacNew (short-term only).

\section{Excluded studies and erroneous data}

Despite the SF-20 Questionnaire being broadly a derivative of the SF-36 and SF-12, we did not include one 
study $^{29}$ in the SF-36 analyses as the questions and scoring algorithms are not sufficiently comparable. One study ${ }^{46}$ described exercise performed as an inpatient prior to randomisation. We included this study as the prerandomisation exercise involved gentle mobilisation only, as opposed to a structured CR exercise intervention, thus fitting with our inclusion criteria. For the same reasons, we excluded a study ${ }^{50}$ in which both groups did complete a structured exercise-based CR intervention prior to randomisation. Following full-text retrieval, we excluded two studies ${ }^{5152}$ which, although aimed to increase participation in physical activity, employed general lifestyle interventions as opposed to exercise-based CR as defined in our protocol.

For one study, ${ }^{41}$ only selected SF-36 variables were reported; physical function domain, and mental component score and physical component score. We were able to include the physical function domain data but were unable to include the mental and physical component score subscales data as the mean values reported were out of range for the measure. Data from another study ${ }^{53}$ reporting the SF-36 could not be accurately extracted from a line-graph, and for another, ${ }^{31}$ could not be metaanalysed, as the way in which the data were reported meant multiple assumptions would have been required. For the EQ-5D, we performed meta-analysis at the shortterm time-point using data from two studies. ${ }^{2734}$ We could not include EQ-5D data from one study, ${ }^{47}$ as all the information required for a meta-analysis was not reported. For another study, ${ }^{49}$ mean values were out of the measurement range for the MacNew Questionnaire, thus, while otherwise the study met our inclusion criteria, the data could not be included. Online supplementary appendix 2 shows how data were extracted and included in the metaanalyses where means or SDs were not explicitly reported.

\section{Sample size, gender, age and study origin}

We included 4890 randomised participants in our analyses. Of the 24 studies, 21 included both male and female participants, 1 study included males only ${ }^{33}$ and 1 study did not specify ${ }^{43}$ the gender of participants recruited (table 1). The mean age of participants in each study was 62 years, range 53-77 years. One study reported an incorrect mean age. ${ }^{49}$ Three studies were conducted in the UK, ${ }^{364042} 9$ elsewhere in Europe 242529303438444547 and 12 outside Europe. ${ }^{27} 31-333537394143464849$

\section{Participant diagnosis of coronary artery disease}

Participant diagnoses, that is, manifestations of CAD, was described in all studies (table 1). Fourteen trials included participants with a range of diagnoses including CAD confirmed with coronary angiography, angina pectoris, myocardial infarction, percutaneous coronary intervention and/or coronaryarterybypass grafting. ${ }^{2729-323437-414447}$ Four studies included participants following myocardial infarction only, 24353646 and one study, angina pectoris only. ${ }^{42}$ Five studies recruited participants 'following coronary artery bypass grafting, 2533434549 and one study 'after percutaneous coronary intervention'. ${ }^{48}$

\section{Treatment received}

Twelve studies included participants who had been revascularised by percutaneous coronary intervention or coronary artery bypass grafting following a recent or past cardiac event ${ }^{24} 273132$ 35-38 40-42 44 (table 1). Five studies recruited participants following percutaneous coronary intervention only 35394648 and five studies recruited participants following coronary artery bypass grafting only. ${ }^{25} 33434549$ It was unclear in two studies whether participants had been revascularised before randomisation and, if so, by which specific procedure. ${ }^{30} 47$

\section{Medication}

Thirteen studies provided a full description of medication (table 1). Six studies referred to medication but provided no specific detail. ${ }^{32} 334247-49$ One study reported beta-blocker usage without reference to other medications. ${ }^{41}$ The remaining four studies did not provide any information about medication. ${ }^{27} 303443$

\section{Recruitment period}

Thirteen studies recruited participants after 31 December 1999 (table 1). In one study, participants were recruited between 1997 and $2000 .{ }^{36}$ On the basis that participant diagnosis, treatment received and co-existing medical therapies indicated 'contemporary' medical care, it was agreed by all reviewers to include this study. This is consistent with the approach used in our previous review of survival. ${ }^{13}$ For the remaining 10 studies, a recruitment period could not be clearly determined from the manuscripts. However, given the description of participant diagnosis, medical treatment, pharmacological therapies and CR interventions, it was agreed by all reviewers that they met our criteria for inclusion.

\section{Content of the interventions}

Intervention content varied considerably between studies (tables 1 and 2). Nineteen studies compared exercise training in combination with additional therapies (education and psychosocial components), two studies compared exercise training as a stand-alone intervention, ${ }^{29}{ }^{41}$ while one study combined exercise and relaxation. ${ }^{43}$ The exercise components of the interventions varied with respect to the setting, training modality, duration, session length, frequency and intensity. The majority of studies incorporated walking and/or cycling as the main exercise modality, delivered for a period ranging from 4 to 12 months, in either an inpatient, home-based or out-patient setting.

\section{Overall effects of interventions}

\section{SF-36 short-term}

We included data from four trials $(\mathrm{N}=560)$ for six SF-36 domains ${ }^{27} 353743$ and data from five trials for the physical function ${ }^{27} 35374143$ and bodily pain ${ }^{27} 35374043$ domains $(\mathrm{N}=596$ and 600) in our meta-analyses (figure 2A). The 


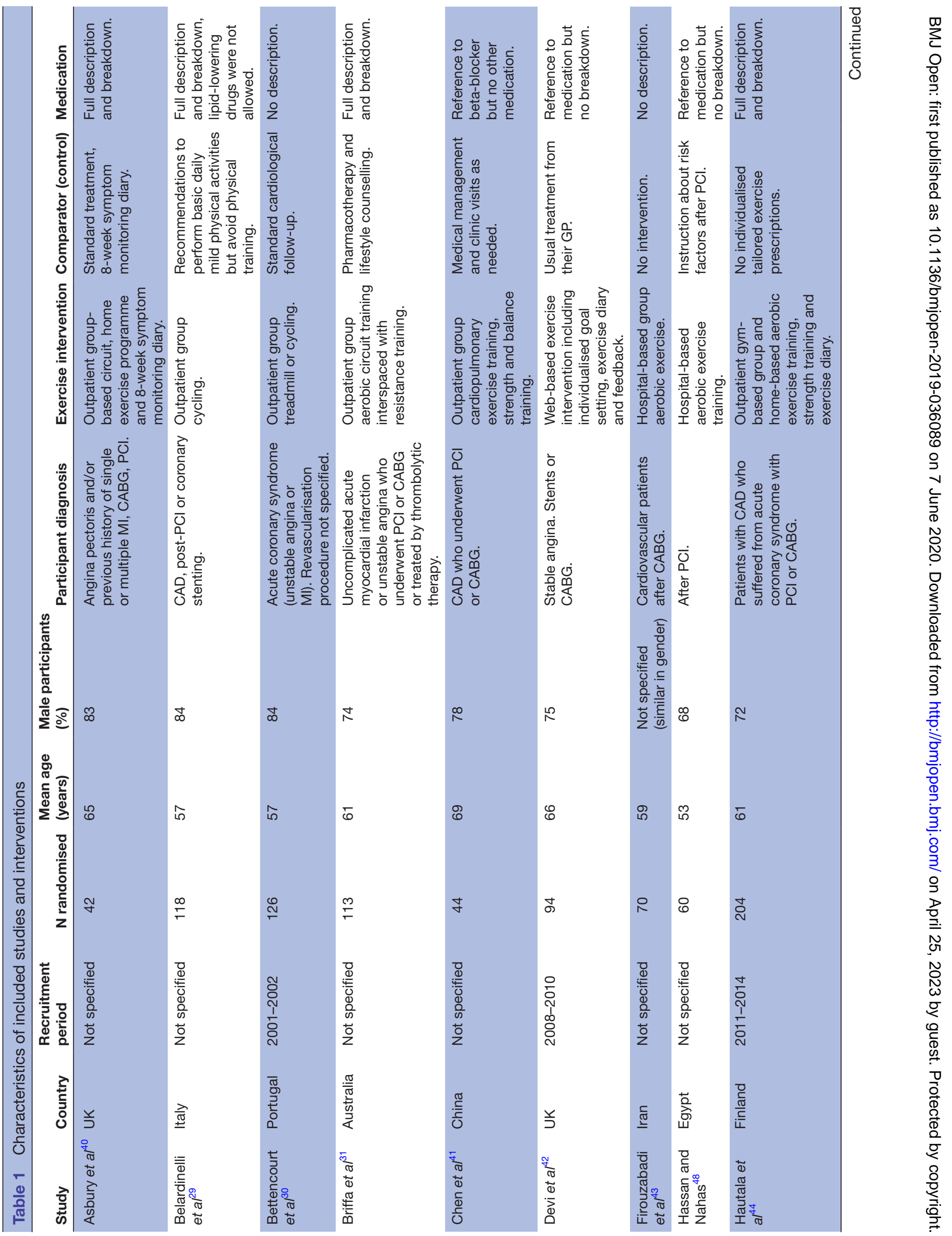




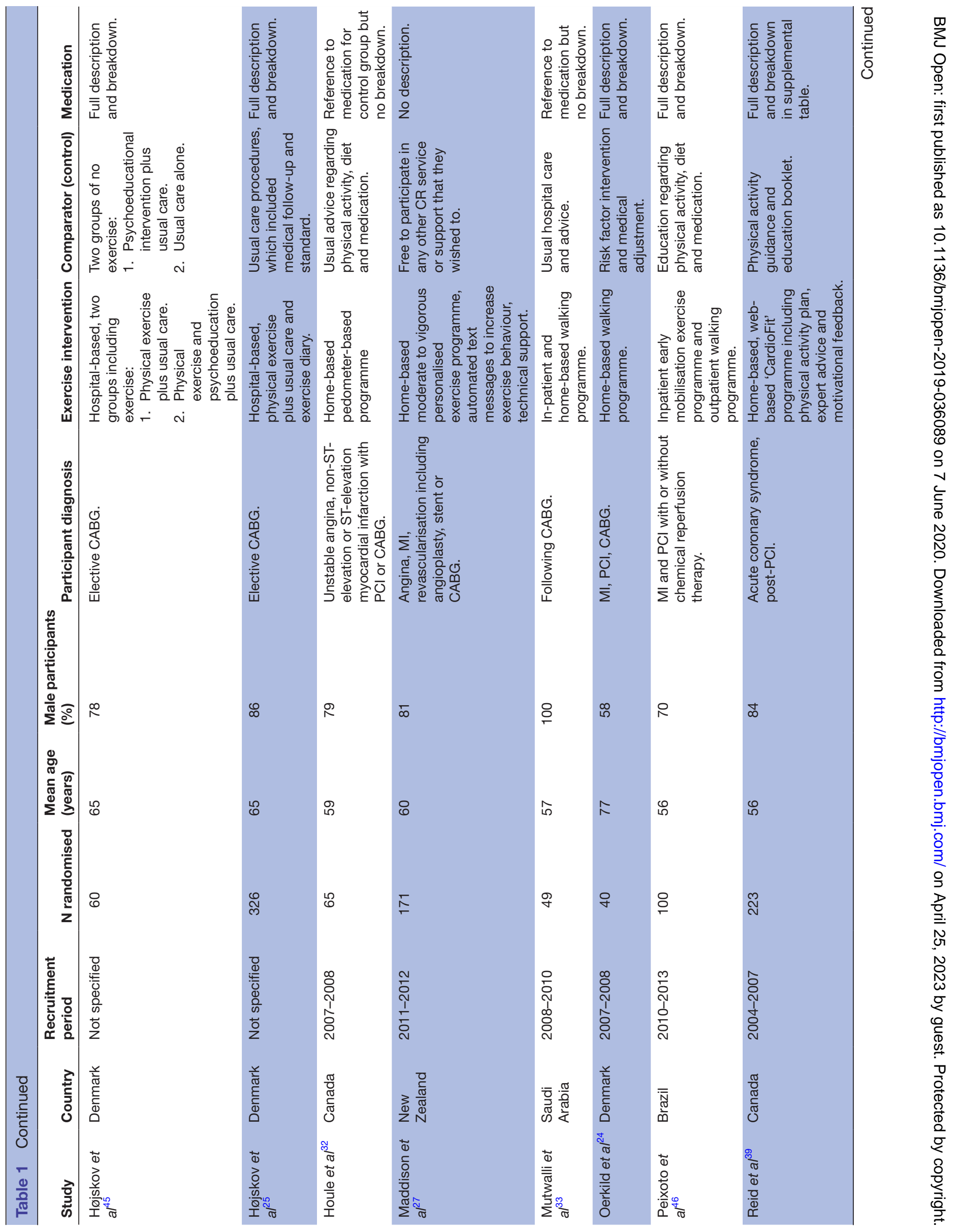




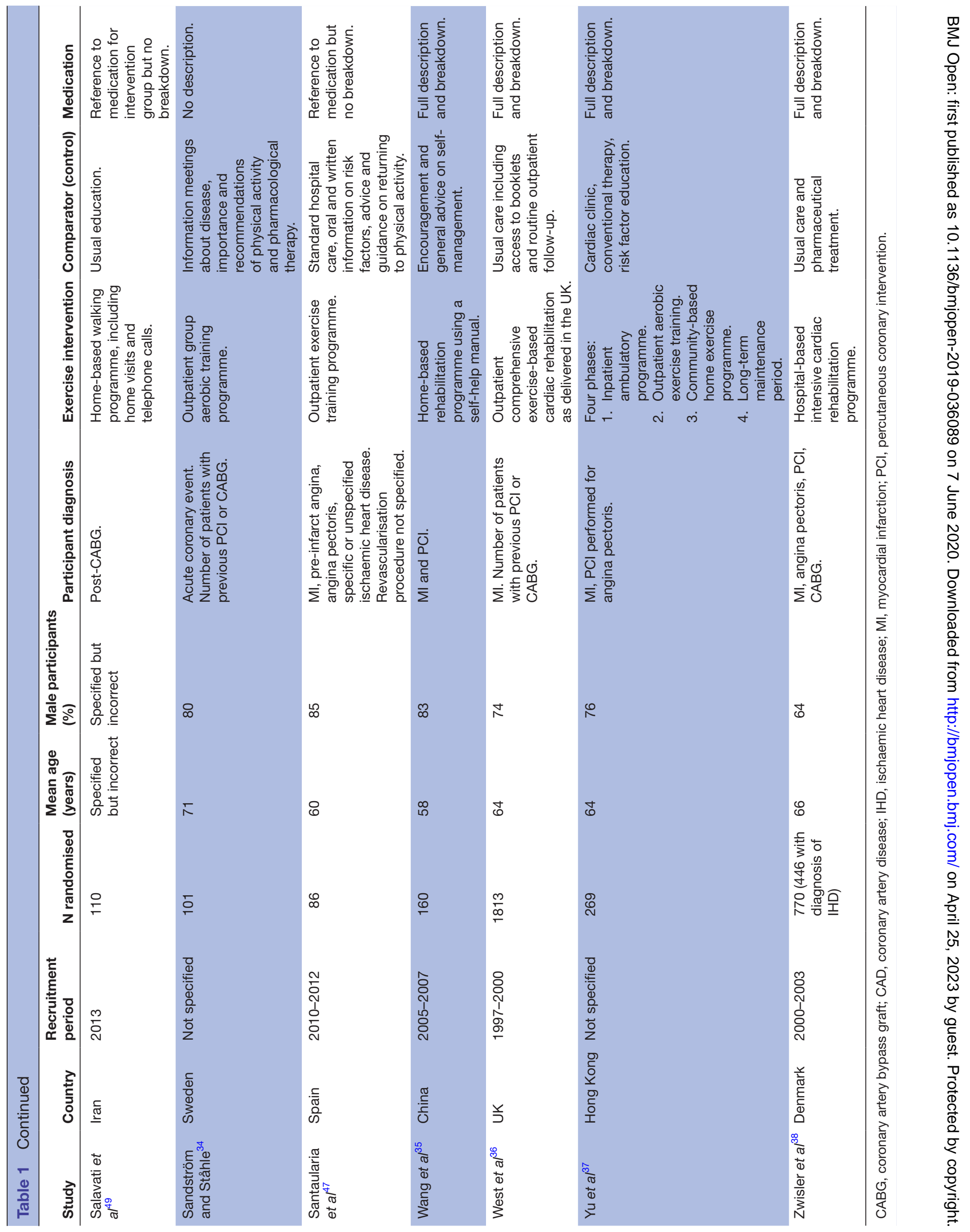




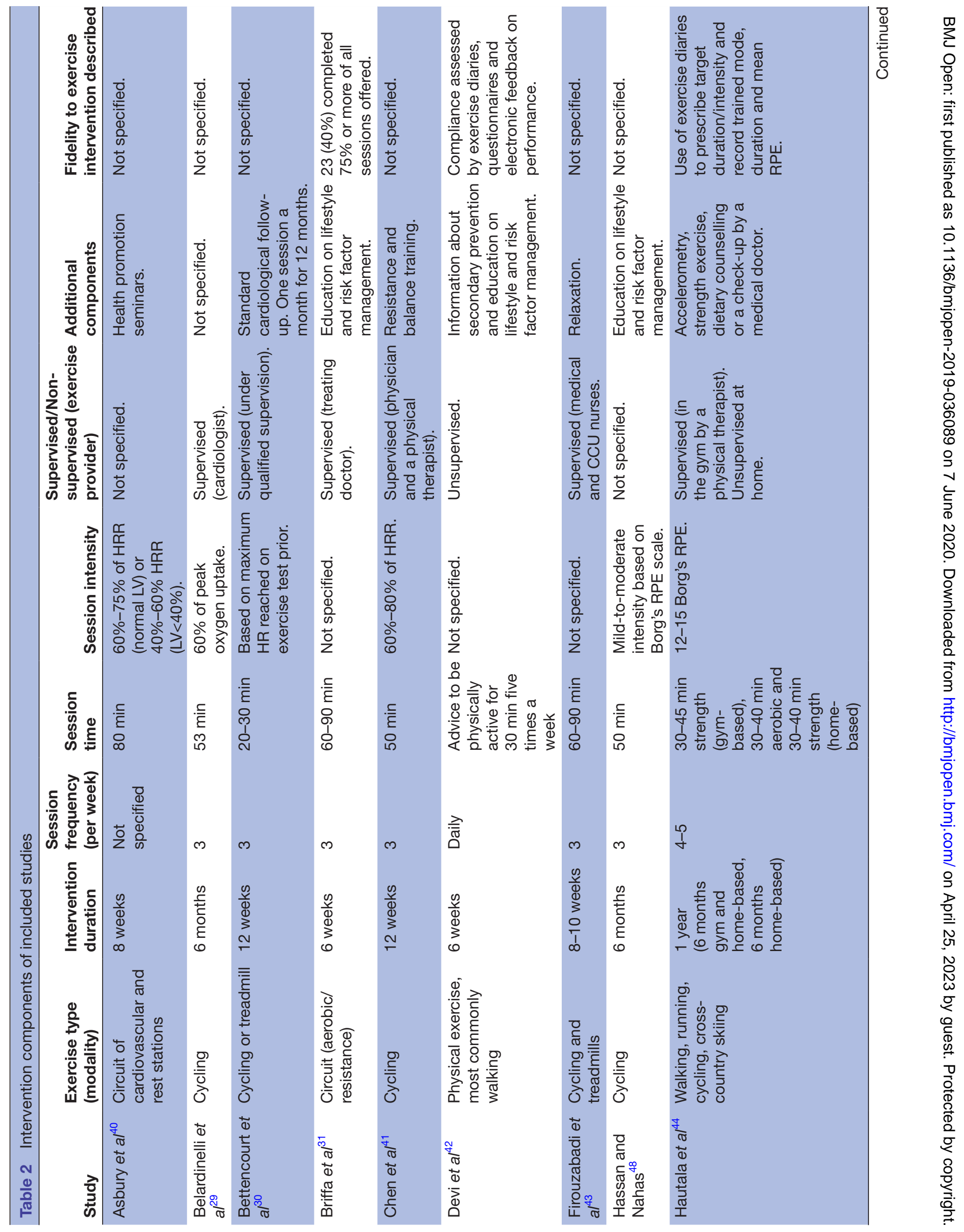




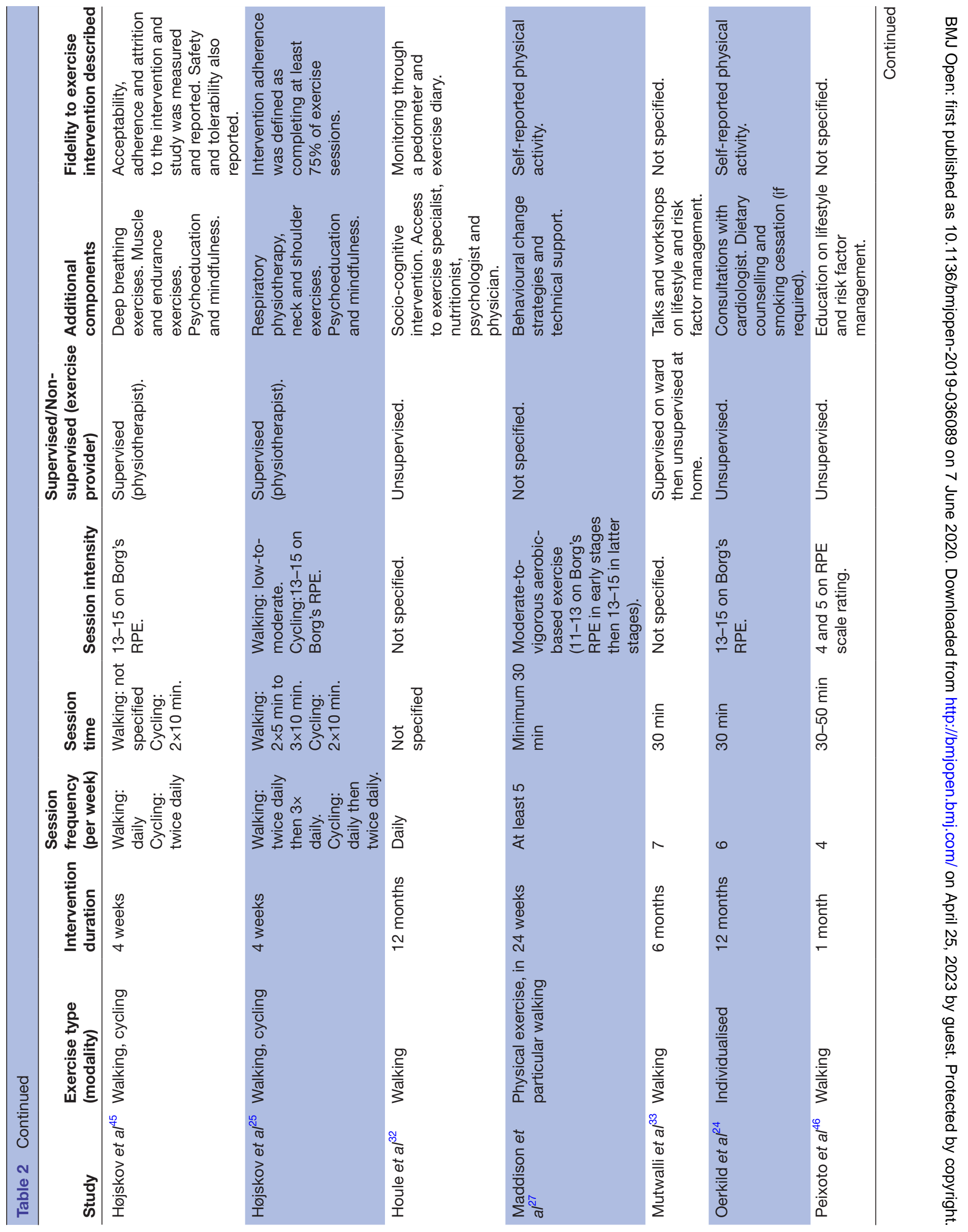




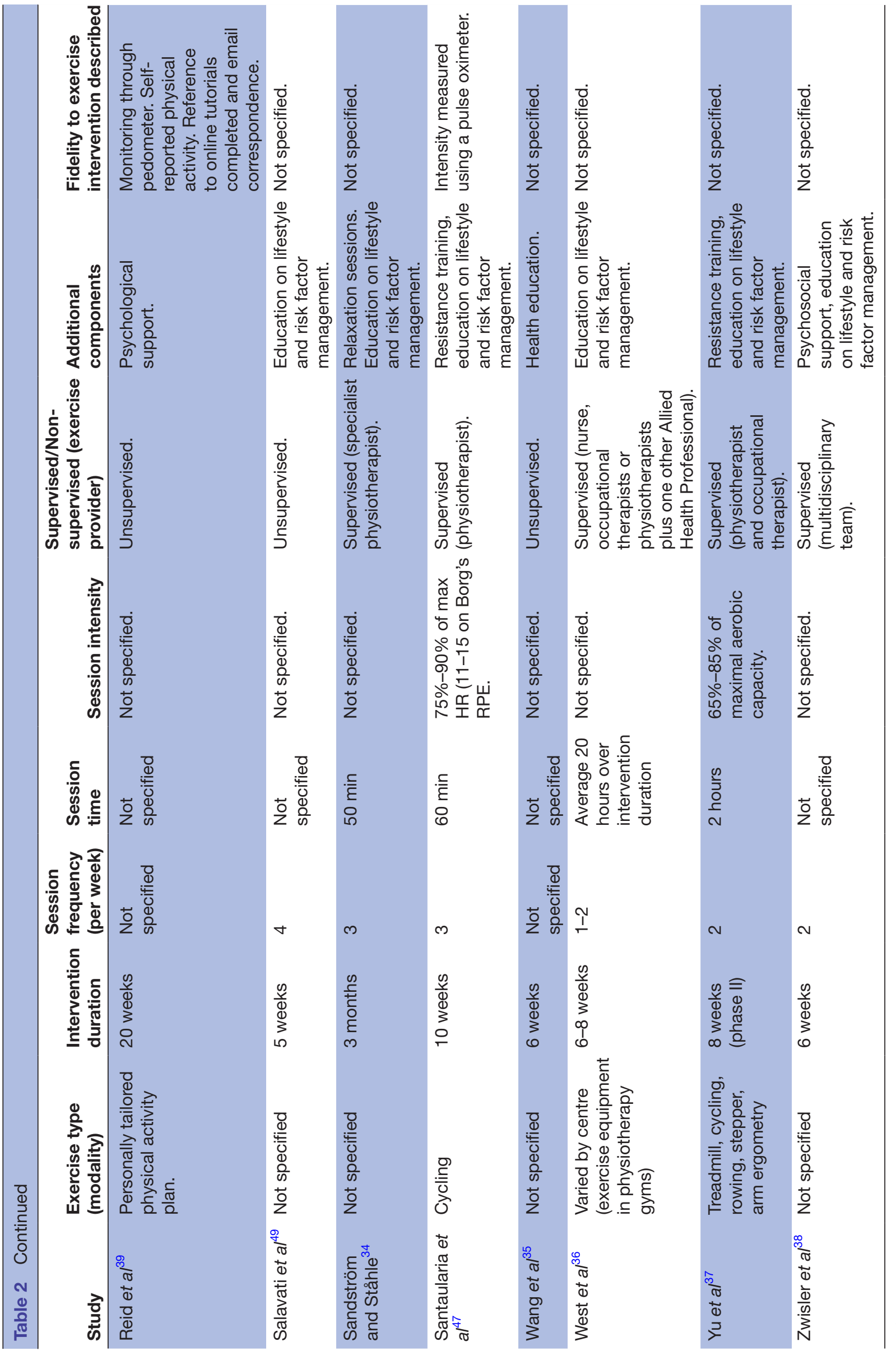


A.

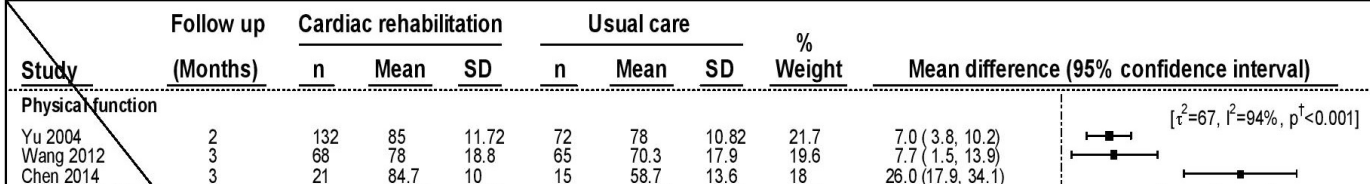
\begin{tabular}{l|l} 
Chen 2014 & 3 \\
Firouzabadi 2014 \\
Maddison 2014
\end{tabular}$\quad \begin{aligned} & 3 \\
& 6\end{aligned}$

All studies Role physical Yu 2004
Wang 2012 Wang 2012 Maddison 2014

All studies

Yu 2004

Asbury 2012

Firouzabadi 2014

Maddison 2014

All studies

Yu 2004
Wang 2012

Firouzabadi 2014

Maddison 2014

All studies

Vitality

Yu 2004
Wang 2012

Firouzabadi 2014

All studies

Social function

Yu 2004

Firouzabadi 2014

Maddison 2014

All studies

Role emotional

Yu 2004

Wang 2012

Maddison 2014

All studies

Mental health

Yu 2004

Wang 2012

Maddison 2014

All studies

$\begin{array}{llllll}85 & 11.72 & 72 & 78 & 10.82 & 21.7 \\ 78 & 18.8 & 65 & 70.3 & 17.9 & 19.6 \\ 84.7 & 10 & 15 & 58.7 & 13.6 & 18 \\ 69.85 & 17.46 & 35 & 47.52 & 16.07 & 18.2 \\ 52.9 & 5.21 & 78 & 51.9 & 5.21 & 22.4\end{array}$

$26.0(17.9,34.1)$
$22.3(1.5,30.2)$
$1.0(-0.7,2.7)$
$12.0(4.4,19.6)$

$26.0(17.9,34.1)$
$22.0(14.5,30.2)$
$12.0(4.4,2.79 .6)$

265

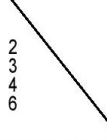

331

$\begin{array}{llllll}78 & 14.65 & 72 & 59 & 21.65 & 267 \\ 69.5 & 43.1 & 65 & 53.4 & 46.3 & 2 \\ 67.85 & 26.48 & 35 & 35.71 & 14.46 & 2 \\ 52.6 & 6.63 & 78 & 50.8 & 6.63 & 27.6\end{array}$

78

26.7
21.2
22.5
27.6

$19.0(13.4,24.6)$

$32.1(22.1,42$.

$1.8(-0.3,3.9)$

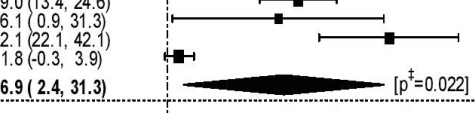

$\left[\tau^{2}=68, l^{2}=92 \%, p^{\dagger}<0.001\right]$

${ }^{\dagger} \mathrm{p}$-value for testing heterogeneity

ours usual care

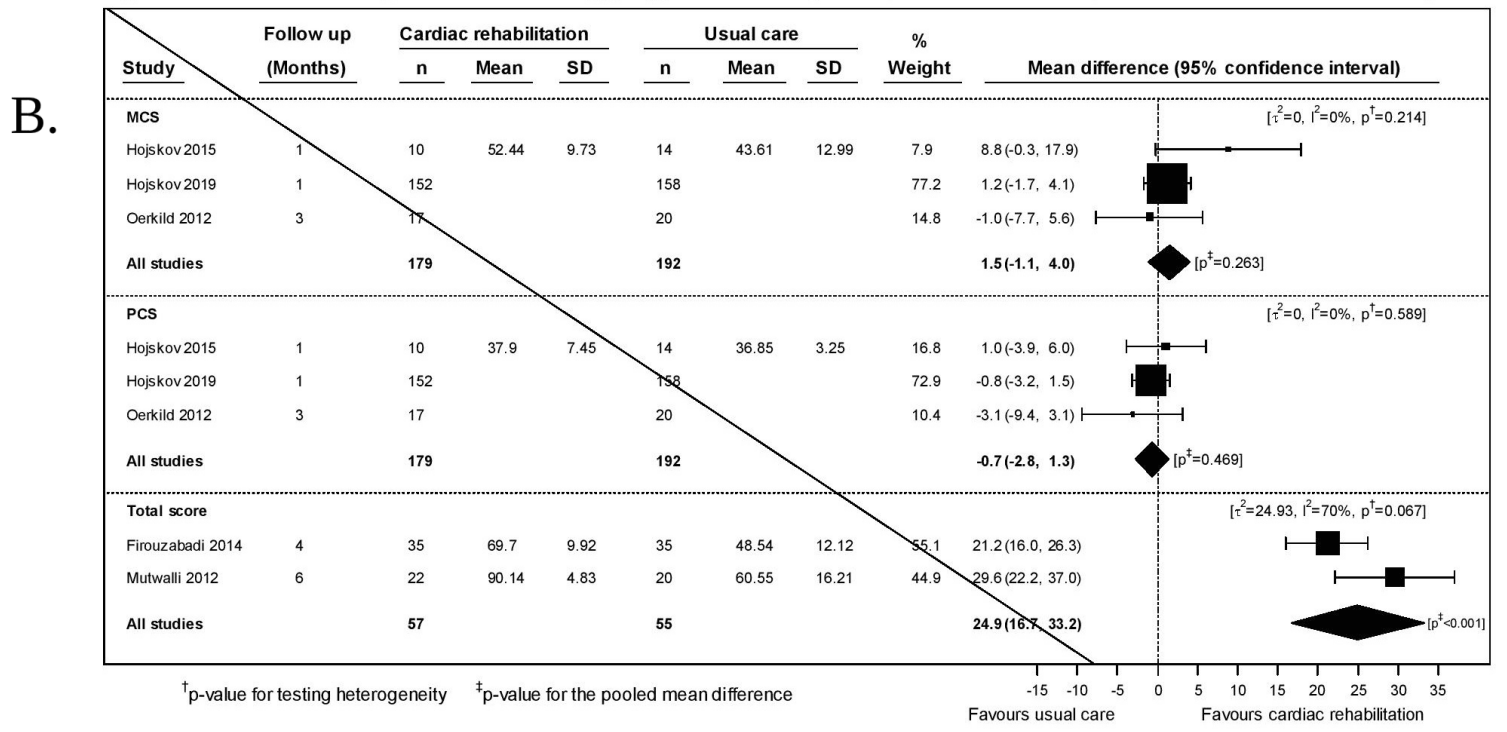

1

Figure 2 (A) Meta-analysis for quality of life (36-Item Short Form Survey Instrument (SF-36) domains) at the short-term timepoint. (B) Meta-analyses of SF-36 aggregate scores (MCS, mental component score; PCS, physical component score and total) at the short-term time-point. 
data were heterogeneous $\left(\mathrm{I}^{2}>80 \%\right.$ for seven of eight domains). Point estimates favoured exercise-based CR in all domains. In six domains, physical function (12.0 (95\% CI 4.4 to 19.6)), role physical (16.9 (2.4 to 31.3)), general health (4.6 (1.9 to 7.2)), vitality (7.6 (1.2 to 14.0$)$ ), social function (10.9 (2.0 to 19.8)) and mental health (4.4 (0.3 to 8.6)), these differences were statistically significant.

We included data from three trials $(\mathrm{N}=371)^{242545}$ that reported the physical and mental component scores of the SF-36. No statistical significant differences were found (figure 2B). We meta-analysed data from two studies $(\mathrm{N}=112)^{33} 43$ reporting an overall SF-36 score. A statistically significant benefit (24.9 (95\% CI 16.7 to 33.2$)$ ) was found.

\section{SF-36 medium-term}

In our meta-analyses, we included data from three trials $(\mathrm{N}=1870)^{36} 3748$ for six SF-36 domains, and data from four trials $(\mathrm{N}=1996)^{30} 363748$ for the general health and vitality domains. The data were less heterogeneous than the short-term data $\left(\mathrm{I}^{2}>80 \%\right.$ for three of eight domains). Point estimates favoured exercise-based CR in all domains. In five domains, physical function (4.0 (95\% CI 0.7 to 7.3$)$ ), role physical (6.9 (0.2 to 13.6$)$ ), general health $(6.0(0.9$ to 11.1$))$, vitality (6.5 (0.6 to 12.3$))$ and social function (6.2 (0.9 to 11.4)), these differences were statistically significant (figure $3 \mathrm{~A}$ ).

We included data from two trials $(\mathrm{N}=478)^{30} 38$ that reported the mental component score of the SF-36, one of which also reported the physical component score $(\mathrm{N}=$ 372) ${ }^{38}$ No statistically significant differences were found (figure 3B).

\section{EuroQol-5D}

We included data from two studies that reported shortterm outcomes for the EQ-5D (N=254) ${ }^{2734}$ The point estimate favoured exercise-based CR but was not statistically significant (figure 4A). No studies reported medium-term outcomes for the EQ-5D.

\section{MacNew Questionnaire}

We included data from three studies that reported shortterm outcomes for the MacNew subscales $(\mathrm{N}=316,316$ and 318). ${ }^{39} 4246$ Two of these also reported an overall score $(\mathrm{N}=242) .{ }^{39}{ }^{46}$ All point estimates favoured exercisebased CR but there were no statistically significant differences (figure 4B).

\section{Other measures}

Eight other QoL measures were each reported by one study. Statistically significant benefits were found in seven out of eight domains of the MOS (Medical Outcomes Study 20-Item Short Form Survey) 20 at 12 months, the EQ-5D mobility subscale at 12 months, the DASI at 4 and 12 months, the Seattle Angina Questionnaire emotional score at 6 weeks, the overall Quality of Life Index-cardiac version III and the same five out of seven of MIDAS subscales at 3 and 12 months (online supplementary appendix 3).

\section{Risk of bias assessment}

We assessed two of the studies included in our metaanalyses as having a low risk of bias $(\mathrm{N}=463),{ }^{25} 27$ nine as having 'some concerns' ( $\mathrm{N}=2493)^{2434-363842454648}$ and the remaining seven as high risk $(\mathrm{N}=671)^{3033} 3739-4143$ (online supplementary appendix 4, figure 5). Methodological issues leading to a classification of high risk of bias related primarily to two domains: (1) deviations from the intended interventions and (2) missing outcome data. For the former, lack of intention-to-treat analysis and inadequate blinding were common issues. For the latter, high loss to follow-up was a common issue.

\section{DISCUSSION}

We performed meta-analyses of 15 short-term and 9 medium-term outcomes. With such a large number of comparisons, some statistically significant findings could be expected due to random chance. Two-thirds of the analyses $(16 / 24)$ were for the eight SF-36 domains. Multiple individual SF-36 domain scores showed statistically significant positive results from exercise-based CR, both in the short-term and medium-term. However, the domains in which a statistically significant effect was observed were different for the short-term and medium-term outcomes. These findings should be interpreted with considerable caution; the quality of the included trials was generally poor and there was substantial statistical heterogeneity. Nevertheless, the overall picture for all domains at both time points favours exercise-based CR, suggesting that there may be an overall benefit on SF-36 domain scores.

The meta-analyses of the SF-36 physical and mental component scores at 12 months, in contrast, did not show any benefit from exercise-based CR. We have for completeness included a meta-analysis of an overall SF-36 score showing a clear benefit from exercise-based CR. The SF-36 overall score is not an accepted metric. ${ }^{54}{ }^{55}$ While these studies met our inclusion criteria, we attach very little weight to this finding because of the non-standard approach to the analysis of the SF-36.

The meta-analysis for the MacNew Questionnaire, a cardiovascular disease-specific outcome, did not find any statistically significant short-term benefit. Nevertheless, for all three domains, the direction of change favoured exercise-based CR. We found no data on the mediumterm or long-term benefits of exercise-based CR on cardiovascular disease-specific QoL. Similarly, the metaanalysis of EQ-5D data found a non-significant difference in favour of exercise-based CR.

A broadly similar pattern was seen in the trials not suitable for meta-analysis, with some statistically significant findings on certain outcomes but with no consistent support for benefit. Taking all of these data into account, our interpretation is that there is some evidence of a beneficial effect of exercise-based CR on QoL in the short-term and insufficient data to comment on the medium-term or long-term benefits. In combination, therefore, undertaking an exercise programme, risk factor modification 


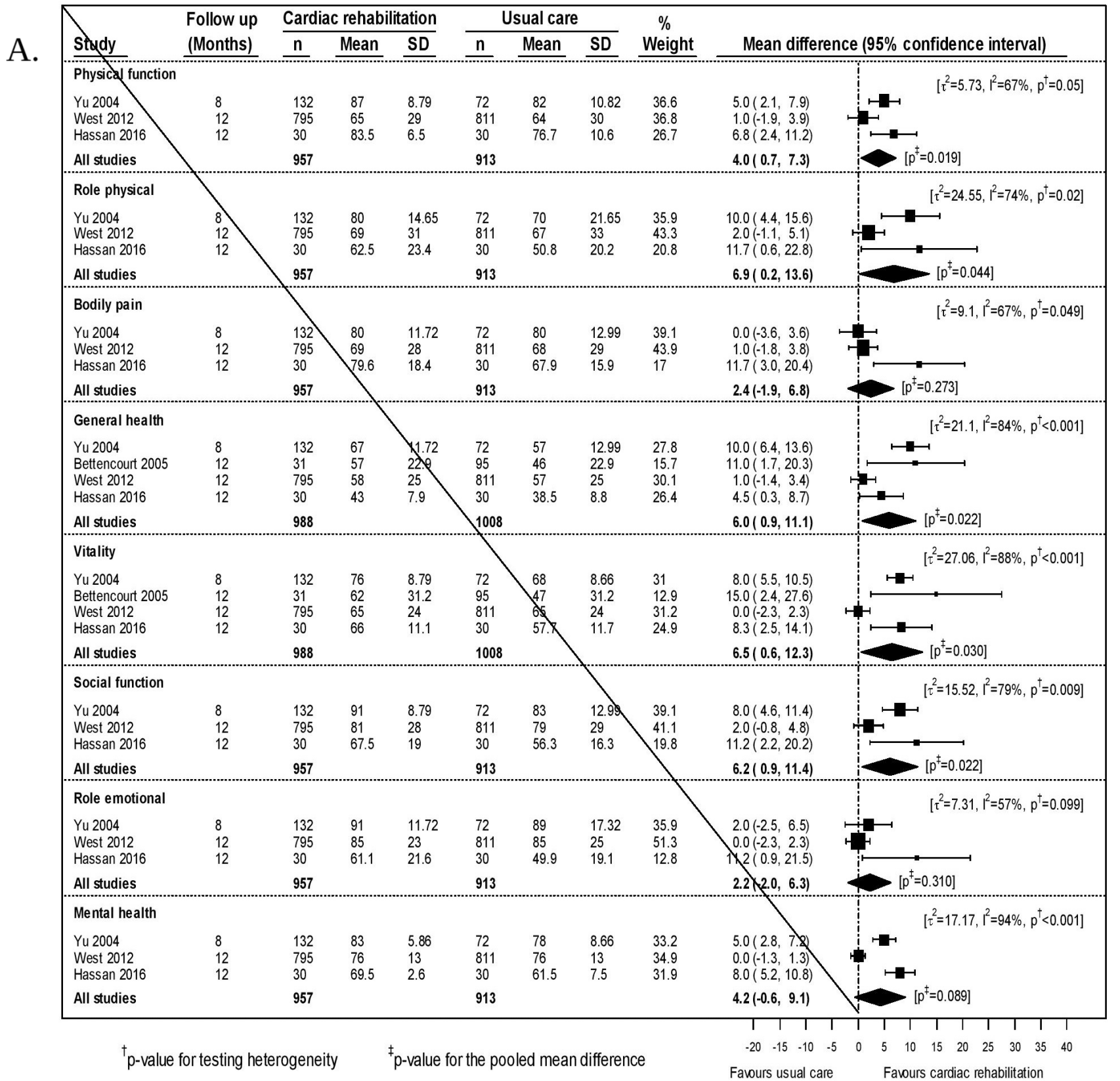

B.

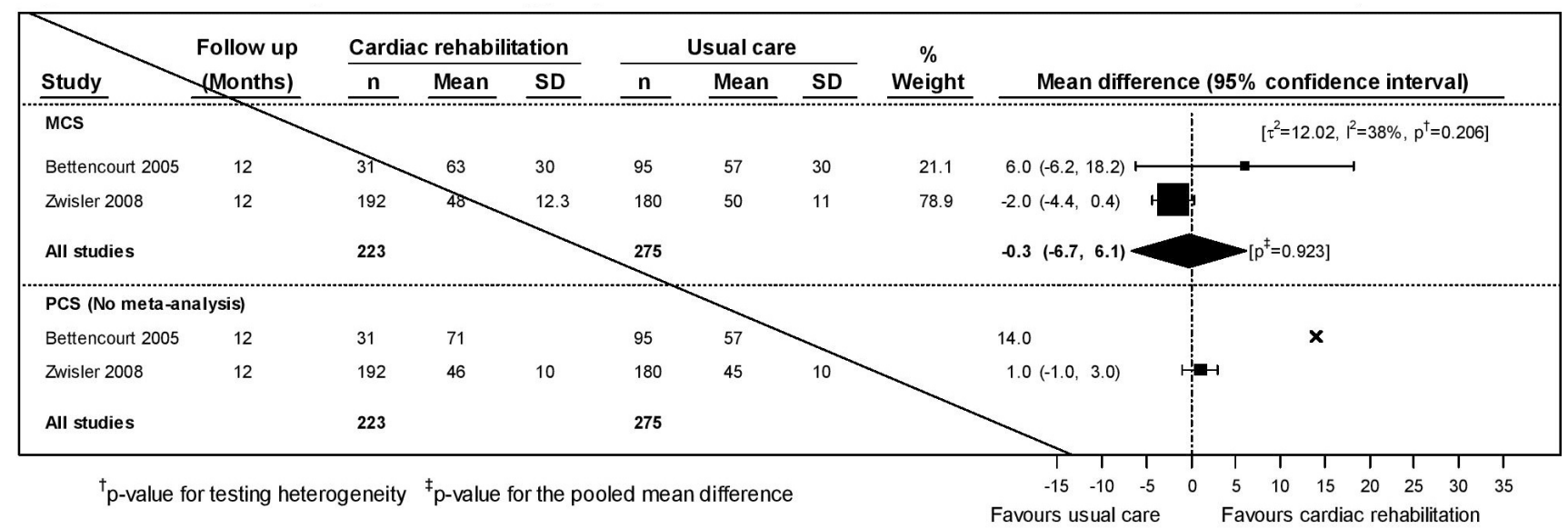

Figure 3 (A) Meta-analysis for quality of life (36-Item Short Form Survey Instrument (SF-36) domains) at the medium-term time-point. (B) Meta-analyses of SF-36 aggregate scores (MCS and PCS) at the medium-term time-point. 
A.

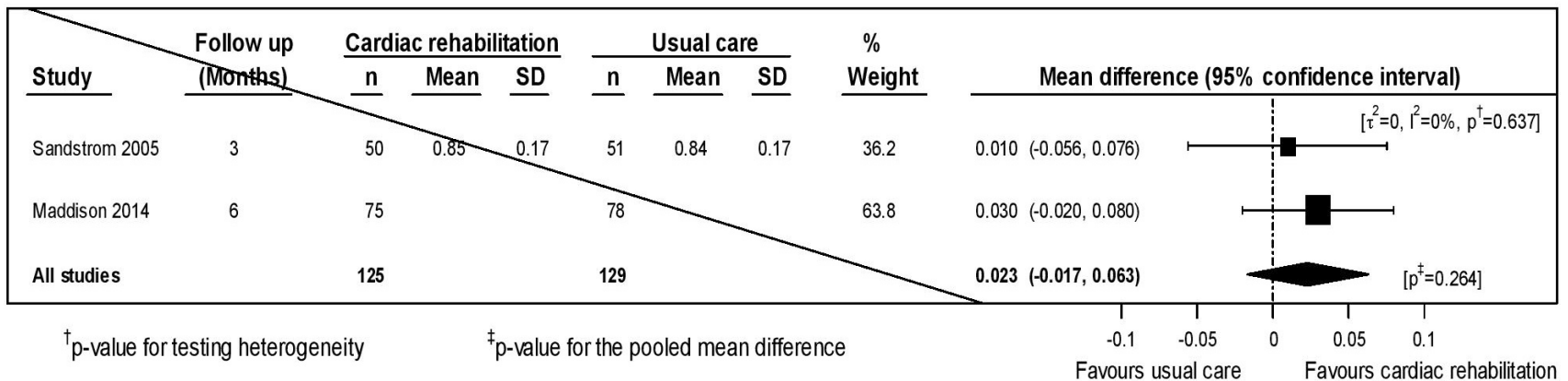

B.

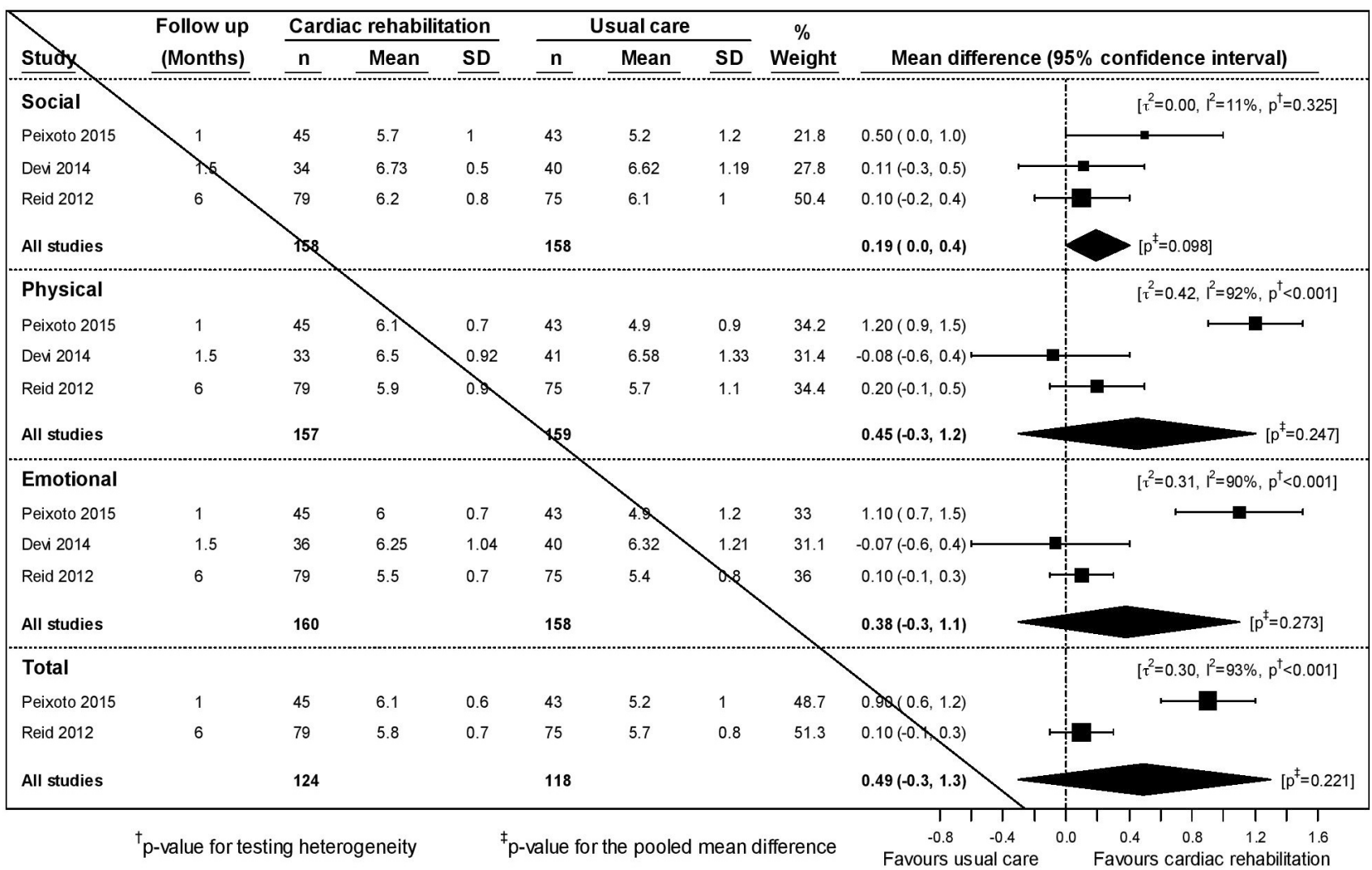

Figure 4 (A) Meta-analysis for EuroQol-5D (EQ-5D) at the short-term time-point. (B) Meta-analysis for quality of life (MacNew) at the short-term time-point.

and behavioural education as part of a comprehensive CR programme may have some impact on individual domains of health-related QoL.

Our observations are limited by the quality of the included studies and the heterogeneity of both the trial participants and the interventions tested. We cannot exclude the possibility that there are subgroups for whom exercise-based CR is effective. To contextualise this observation, data from our previous review of mortality ${ }^{13}$ in exercise-based CR should be considered (mortality was not assessed in the current review). The review was criticised for not considering the potentially greater benefit for those who adhere to treatment. ${ }^{56}$ Since the overall effect on mortality in our previous review was zero, any reduced mortality in the subgroup that adhered, would inevitably mean an equal increase in mortality in participants who did not adhere. In contrast, given the positive effect of exercise-based CR on QoL in the current analyses, it is plausible that poor adherence to the intervention is attenuating the benefits. If there is a zero effect in those who do not adhere, there may be a worthwhile effect in those who do adhere. None of our included studies presented an analysis that would allow the effect size in adherent participants to be estimated appropriately. ${ }^{57}$ The approach of comparing outcomes in the adherent group with overall outcomes in the control group used 


\section{As percentage (intention-to-treat)}

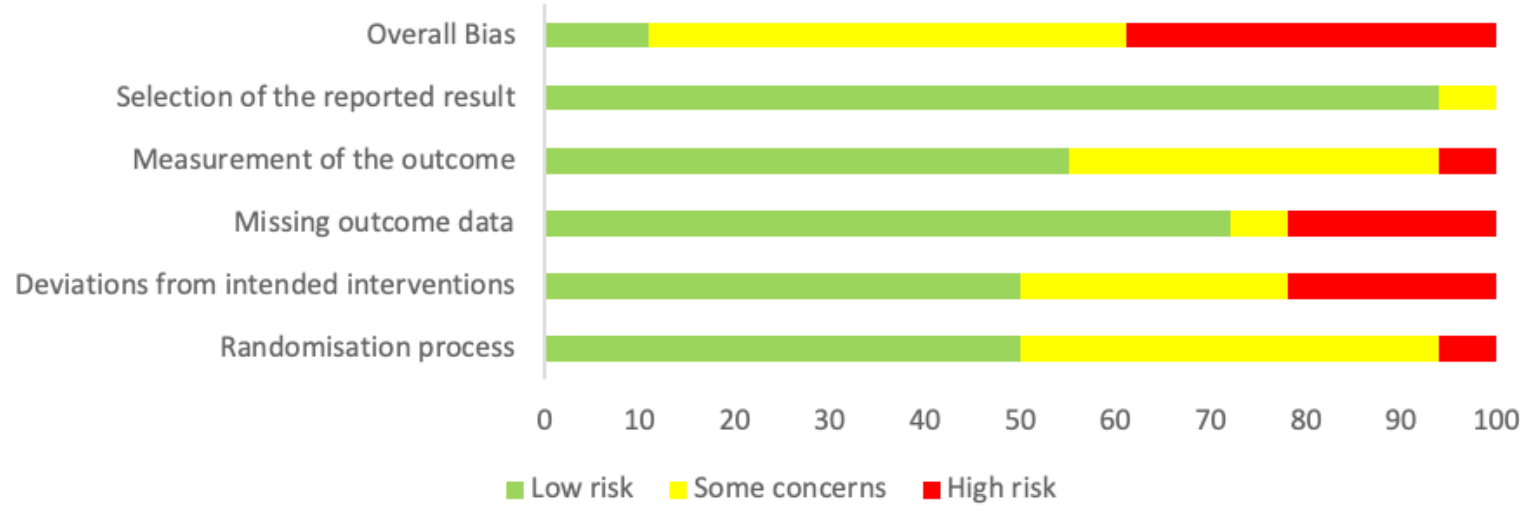

Figure 5 Risk of bias assessment. Does exercise-based cardiac rehabilitation improve quality of life in coronary artery disease? A contemporary systematic review and meta-analysis.

by some authors ${ }^{25}$ is potentially misleading. An appropriate approach, such as a complier average causal effect analysis, would adjust for non-compliance, thus providing more reliable results.

Our previous review ${ }^{13}$ has been criticised for including the RAMIT (Rehabilitation After Myocardial Infarction Trial) trial. ${ }^{36}$ Other recent reviews ${ }^{20}$ using the same recruitment period criteria as us, excluded the RAMIT trial whose recruitment straddled the end of 1999. In a post hoc sensitivity analysis we excluded RAMIT data. It did not materially affect our conclusions (online supplementary appendix 5). For completeness, we also provide our previous mortality analysis with RAMIT excluded (online supplementary appendix 6). Again, this does not materially change our previous conclusions.

Post hoc, to inform a discussion on the clinical relevance of our findings, we searched unsuccessfully for established values of clinically important between-group differences, following CR, for the outcomes included in our meta-analyses. For the SF-36, the minimal clinically important within-person change has been reported as an increase in any domain score of three to five points. ${ }^{58}$ All of our statistically significant differences in the SF-36 domains met this threshold. However, caution is strongly advised with this arbitrary value as the clinically important within-person change varies considerably, dependent on diagnosis and duration and severity of disease, among other confounders. ${ }^{59} 60$ Therefore, we looked for values of a clinically important within-person change in SF-36 domains following CR. On the basis that an improvement equating to half of the within-person change can be considered a worthwhile outcome for an appreciable number of people ${ }^{61}$ we set this as a criterion for a clinically important between-group difference. Usefully, for our current purpose, a heart disease expert consensus ${ }^{62}$ suggested SF-36 domain-specific changes that should be considered minimal, moderate and large for an individual. Minimal changes ranged from 15 to 25 points, and moderate changes from 25 to 50 points (online supplementary appendix 7 ). Using this approach, the only clinically important differences in the SF-36 domains were the short-term effects on 'physical function' and 'role physical'. These are above, or close to half of, the consensus values for a moderate change. No other point estimates met the criteria for a clinically important change.

For the MacNew Questionnaire, a within-person change of 0.5 points for any specific domain or the overall score has been proposed. ${ }^{63-65}$ Using the same approach, we would set a between-group difference of 0.25 points as a clinically important benefit. Although none of the analyses was statistically significant, the point estimates for physical and emotional subscales and the overall score are consistent with a clinically important short-term benefit on the MacNew Questionnaire.

\section{Strengths and limitations}

We identified 24 studies, 18 of which we could include in meta-analyses. Due to wider inclusion criteria in terms of time-points for outcome reporting, this is $>14$ studies identified (8 meta-analysed) in another recent review. ${ }^{20}$ Also, our search date was more recent, and we included studies testing exercise-based CR interventions in all manifestations of CAD rather than just those with acute coronary syndrome \pm revascularisation, angina or angiographically documented CAD. We did, however, exclude one study ${ }^{53}$ from our meta-analyses that was included by the previous authors. We were unable to extract data from the line graph, and values presented in the text were not between groups differences so could not be used. We also differ from the other recent review in our interpretation of a clinically important benefit for the SF-36. This is because we used consensus values for minimal and moderate change $^{62}$ to define clinical importance, rather than the smallest measurable change in an SF-36 domain. We would interpret their findings as showing that there were 
no clinically important benefits on any SF-36 domains and that only role physical achieved a minimal benefit.

Given the heterogeneity and paucity of data included in our review, strengths and weaknesses should be considered. We performed rigorous and transparent systematic review, with meta-analysis where possible. Where there was any doubt as to data compatibility, we opted to exclude studies from the analysis, helping to ensure the integrity of the results. We included only data from studies recruiting after 31 December 1999 to ensure that findings were applicable in the era of contemporary medical care. Defining the era of contemporary medical care can be problematic due to difficulties in identifying exactly when data were collected for each trial, and the nature of medical practice at the time. Therefore, it is not possible to be certain that included and excluded trials exactly match our criteria for what constitutes contemporary medical care. However, meticulous examination of each trial provides a high level of confidence that the most appropriate studies have been included.

Our findings are limited by a number of inconsistencies in the CR literature and data. While baseline data were always collected prior to randomisation, follow-up data were reported at varying times postrandomisation. Our short-term data included studies reporting their first follow-up at anything between 1 and 6 months. Equally, our medium-term data covered studies reporting between 8 and 12 months. Data were too scarce and heterogeneous to assess time-points more accurately. It is also worth noting that only 5/24 analyses in our review included studies using CAD-specific QoL measures. It is possible that generic QoL instruments are insufficiently sensitive to detect change in people with CAD. Diseasespecific tools are more likely to accurately reflect QoL in this population.

Exercise interventions and other core components of comprehensive $\mathrm{CR}$ varied widely in their composition and delivery, and these may have fallen short of what would be considered 'optimal' or 'gold standard' care. Equally, usual care was inconsistent which may dilute any benefit associated with exercise-based CR. Furthermore, the overall quality of studies included in our meta-analyses was poor, with the majority scoring 'some concerns' or 'high' on the risk of bias assessment. Numerous sources of potential bias were identified including poor reporting of key methodological information such as randomisation, blinding and statistical analyses.

Data reporting in some studies is a potential source of bias. First, results from one study ${ }^{46}$ showed a vastly superior improvement in MacNew QoL scores compared with others ${ }^{39} 42$ in the short-term analysis. Second, one study ${ }^{33}$ reported only the total score for the SF-36 at 6 months; this is not a validated or recommended measure. ${ }^{54}{ }^{55}$ Third, for one study, ${ }^{38}$ we only included $\mathrm{n}=372(58 \%)$ participants who had IHD at 12 months follow-up, however, the SF-36 values included a proportion of participants with heart failure $(12 \%)$ or at high risk of ischaemic heart disease $(30 \%)$. However, this only affected the medium-term data for SF-36 MCS and PCS, as these were the only data reported in the trial. Finally, we could not perform a meta-analysis for the SF-36 PCS at 12 months in one study ${ }^{30}$ as an exact $p$ value was not provided.

\section{CONCLUSIONS}

For people with CAD participating in exercise-based CR, our meta-analyses show statistically significant improvements in multiple individual SF-36 domain scores, but only $2 / 24$ comparisons (both short-term outcomes) can be deemed clinically important. Exercise-based CR shows promise as an approach to improve QoL for people with CAD. However, the contemporary data presented in this review are insufficient to support its routine use. Given the critical importance of QoL to people living with longterm conditions, future research should optimise CR programmes to target improvement in QoL domains.

\section{Twitter Gordon McGregor @HIITorMISSUK}

Acknowledgements The authors would like to thank Petra Meeson, Specialist Medical Librarian, UHCW NHS Trust for her expert assistance with database searching.

Contributors Conceptualisation: MU and GMcG; methodology: GMcG, PK, RP and $\mathrm{MU}$; formal analysis: PK; investigation: GMcG, RP and MU; data curation: GMcG and RP; supervision: MU; writing —original draft preparation: GMcG; writing—review and editing: GMcG, RP, PK and MU.

Funding The authors have not declared a specific grant for this research from any funding agency in the public, commercial or not-for-profit sectors.

Competing interests MU was Chair of the NICE accreditation advisory committee until March 2017 for which he received a fee. He is chief investigator or coinvestigator on multiple previous and current research grants from the UK National Institute for Health Research, Arthritis Research UK and is a co-investigator on grants funded by the Australian NHMRC. He is co-applicant on two studies of cardiopulmonary rehabilitation. He is an NIHR Senior Investigator. He has received travel expenses for speaking at conferences from the professional organisations hosting the conferences. He is a director and shareholder of Clinvivo Ltd that provides electronic data collection for health services research. He is part of an academic partnership with Serco Ltd related to return to work initiatives. He is a coinvestigator on a study receiving support in kind from Stryker Ltd. He has accepted honoraria for teaching/lecturing from CARTA \& Sterling Events. He is an editor of the NIHR journal series, and a member of the NIHR Journal Editors Group, for which he receives a fee. GM is a practising exercise physiologist. He is chief investigator and co-investigator on multiple studies of cardiopulmonary rehabilitation funded by NIHR and BHF. RP is a practising exercise physiologist.

Patient and public involvement Patients and/or the public were not involved in the design, conduct, reporting or dissemination plans of this research.

Patient consent for publication Not required.

Provenance and peer review Not commissioned; externally peer reviewed.

Data availability statement All data relevant to the study are included in the article or uploaded as supplementary information. Data are available in supplementary material.

Open access This is an open access article distributed in accordance with the Creative Commons Attribution Non Commercial (CC BY-NC 4.0) license, which permits others to distribute, remix, adapt, build upon this work non-commercially, and license their derivative works on different terms, provided the original work is properly cited, appropriate credit is given, any changes made indicated, and the use is non-commercial. See: http://creativecommons.org/licenses/by-nc/4.0/.

ORCID iD

Gordon McGregor http://orcid.org/0000-0001-8963-9107 


\section{REFERENCES}

1 World Health Organisation. Global health estimates 2016: disease burden by cause, age, sex, by country and by region, 2000-2016. Geneva: World Health Organization, 2018.

2 Keeley EC, Boura JA, Grines CL. Primary angioplasty versus intravenous thrombolytic therapy for acute myocardial infarction: a quantitative review of 23 randomised trials. Lancet 2003;361:13-20.

3 Gaziano TA, Bitton A, Anand S, et al. Growing epidemic of coronary heart disease in low- and middle-income countries. Curr Probl Cardiol 2010;35:72-115.

4 British Heart Foundation. Cardiovascular Disease Statistics - BHF UK Factsheet. London, England, 2018.

5 NICE. Secondary prevention after a myocardial infarction. NICE quality standard [QS99]., 2015. Available: https://www.nice.org.uk/ guidance/qs99

6 NICE. Cardiac rehabilitation after myocardial infarction, 2018. Available: https://pathways.nice.org.uk/pathways/myocardialinfarction-rehabilitation-and-preventing-further-cardiovasculardisease/cardiac-rehabilitation-after-myocardial-infarction

7 Piepoli MF, Hoes AW, Agewall S, et al. 2016 European guidelines on cardiovascular disease prevention in clinical practice: The sixth joint task force of the European society of cardiology and other societies on cardiovascular disease prevention in clinical practice (constituted by representatives of 10 societies and by invited experts) developed with the special contribution of the European association for cardiovascular prevention \& rehabilitation (EACPR). Eur Heart $J$ 2016;37:2315-81.

8 Valkeinen $\mathrm{H}$, Aaltonen $\mathrm{S}$, Kujala UM. Effects of exercise training on oxygen uptake in coronary heart disease: a systematic review and meta-analysis. Scand J Med Sci Sports 2010;20:545-55.

9 Anderson L, Thompson DR, Oldridge N, et al. Exercise-based cardiac rehabilitation for coronary heart disease. Cochrane Database Syst Rev 2016:CD001800.

10 Heran BS, Chen JM, Ebrahim S, et al. Exercise-based cardiac rehabilitation for coronary heart disease. Cochrane Database Syst Rev 2011:CD001800.

11 Kabboul NN, Tomlinson G, Francis TA, et al. Comparative effectiveness of the core components of cardiac rehabilitation on mortality and morbidity: a systematic review and network metaanalysis. J Clin Med 2018;7:514.

12 Xia T-L, Huang F-Y, Peng Y, et al. Efficacy of different types of Exercise-Based cardiac rehabilitation on coronary heart disease: a network meta-analysis. J Gen Intern Med 2018;33:2201-9.

13 Powell R, McGregor G, Ennis S, et al. Is exercise-based cardiac rehabilitation effective? A systematic review and meta-analysis to re-examine the evidence. BMJ Open 2018;8:e019656.

14 The World Health Organization quality of life assessment (WHOQOL): position paper from the World Health Organization. Soc Sci Med 1995;41:1403-9.

15 Mercieca-Bebber R, King MT, Calvert MJ, et al. The importance of patient-reported outcomes in clinical trials and strategies for future optimization. Patient Relat Outcome Meas 2018;9:353-67.

16 Francis T, Kabboul N, Rac V, et al. The effect of cardiac rehabilitation on health-related quality of life in patients with coronary artery disease: a meta-analysis. Can J Cardiol 2019;35:352-64.

17 Long L, Anderson L, Dewhirst AM, et al. Exercise-based cardiac rehabilitation for adults with stable angina. Cochrane Database Syst Rev 2018;2:CD012786.

18 Zheng X, Zheng Y, Ma J, et al. Effect of exercise-based cardiac rehabilitation on anxiety and depression in patients with myocardial infarction: a systematic review and meta-analysis. Heart Lung 2019;48:1-7.

19 Resurrección DM, Moreno-Peral P, Gómez-Herranz M, et al. Factors associated with non-participation in and dropout from cardiac rehabilitation programmes: a systematic review of prospective cohort studies. Eur J Cardiovasc Nurs 2019;18:38-47.

20 Candelaria D, Randall S, Ladak L, et al. Health-related quality of life and exercise-based cardiac rehabilitation in contemporary acute coronary syndrome patients: a systematic review and meta-analysis. Qual Life Res 2020;29:579-92.

21 Wood D, De Backer G, Faergeman O, et al. Prevention of coronary heart disease in clinical practice: recommendations of the second joint Task force of European and other societies on coronary prevention. Atherosclerosis 1998;140:199-270.

22 Schwarzer G. Meta: an R package for meta-analysis, 2007: 40-5.

23 Team RC. R: a language and environment for statistical computing. Vienna, Austria: R Foundation for Statistical Computing, 2018. https://www.R-project.org/.

24 Oerkild B, Frederiksen M, Hansen JF, et al. Home-based cardiac rehabilitation is an attractive alternative to no cardiac rehabilitation for elderly patients with coronary heart disease: results from a randomised clinical trial. BMJ Open 2012;2:e001820.

25 Højskov IE, Moons P, Egerod I, et al. Early physical and psychoeducational rehabilitation in patients with coronary artery bypass grafting: a randomized controlled trial. J Rehabil Med 2019;51:136-43.

26 Viechtbauer W. Conducting meta-analyses in $R$ with the metafor Package. J Stat Softw 2010;36:1-48.

27 Maddison R, Pfaeffli L, Whittaker R, et al. A mobile phone intervention increases physical activity in people with cardiovascular disease: results from the heart randomized controlled trial. Eur J Prev Cardiol 2015;22:701-9.

28 Cumpston M, Li T, Page MJ, et al. Updated guidance for trusted systematic reviews: a new edition of the Cochrane Handbook for systematic reviews of interventions. Cochrane Database Syst Rev 2019;10:ED000142.

29 Belardinelli R, Paolini I, Cianci G, et al. Exercise training intervention after coronary angioplasty: the ETICA trial. J Am Coll Cardiol 2001;37:1891-900.

30 Bettencourt N, Dias C, Mateus P, et al. Impact of cardiac rehabilitation on quality of life and depression after acute coronary syndrome. Rev Port Cardiol 2005;24:687-96.

31 Briffa TG, Eckermann SD, Griffiths AD, et al. Cost-effectiveness of rehabilitation after an acute coronary event: a randomised controlled trial. Med J Aust 2005;183:450-5.

32 Houle J, Doyon O, Vadeboncoeur N, et al. Effectiveness of a pedometer-based program using a socio-cognitive intervention on physical activity and quality of life in a setting of cardiac rehabilitation. Can J Cardiol 2012;28:27-32.

33 Mutwalli HA, Fallows SJ, Arnous AA, et al. Randomized controlled evaluation shows the effectiveness of a home-based cardiac rehabilitation program. Saudi Med J 2012;33:152-9.

34 Sandström L, Ståhle A. Rehabilitation of elderly with coronary heart disease - improvement in quality of life at a low cost. Adv Physiother 2005;7:60-6.

35 Wang W, Chair SY, Thompson DR, et al. Effects of home-based rehabilitation on health-related quality of life and psychological status in Chinese patients recovering from acute myocardial infarction. Heart Lung 2012;41:15-25.

36 West RR, Jones DA, Henderson AH. Rehabilitation after myocardial infarction trial (RAMIT): multi-centre randomised controlled trial of comprehensive cardiac rehabilitation in patients following acute myocardial infarction. Heart 2012;98:637-44.

37 Yu C-M, Lau C-P, Chau J, et al. A short course of cardiac rehabilitation program is highly cost effective in improving longterm quality of life in patients with recent myocardial infarction or percutaneous coronary intervention. Arch Phys Med Rehabil 2004;85:1915-22.

38 Zwisler A-DO, Soja AMB, Rasmussen S, et al. Hospital-based comprehensive cardiac rehabilitation versus usual care among patients with congestive heart failure, ischemic heart disease, or high risk of ischemic heart disease: 12-month results of a randomized clinical trial. Am Heart J 2008;155:1106-13.

39 Reid RD, Morrin LI, Beaton LJ, et al. Randomized trial of an internetbased computer-tailored expert system for physical activity in patients with heart disease. Eur J Prev Cardiol 2012;19:1357-64.

40 Asbury EA, Webb CM, Probert $\mathrm{H}$, et al. Cardiac rehabilitation to improve physical functioning in refractory angina: a pilot study. Cardiology 2012;122:170-7.

41 Chen $\mathrm{C}-\mathrm{H}$, Chen $\mathrm{Y}-\mathrm{J}$, Tu H-P, et al. Benefits of exercise training and the correlation between aerobic capacity and functional outcomes and quality of life in elderly patients with coronary artery disease. Kaohsiung J Med Sci 2014;30:521-30.

42 Devi R, Powell J, Singh S. A web-based program improves physical activity outcomes in a primary care angina population: randomized controlled trial. J Med Internet Res 2014;16:e186.

43 Firouzabadi MG, Sherafat A, Vafaeenasab M. Effect of physical activity on the life quality of coronary artery bypass graft patients. $J$ Med Life 2014;7:260-3.

44 Hautala AJ, Kiviniemi AM, Mäkikallio T, et al. Economic evaluation of exercise-based cardiac rehabilitation in patients with a recent acute coronary syndrome. Scand J Med Sci Sports 2017;27:1395-403.

45 Højskov IE, Moons P, Hansen NV, et al. Early physical training and psycho-educational intervention for patients undergoing coronary artery bypass grafting. The SheppHeart randomized $2 \times 2$ factorial clinical pilot trial. Eur J Cardiovasc Nurs 2016;15:425-37.

46 Peixoto TCA, Begot I, Bolzan DW, et al. Early exercise-based rehabilitation improves health-related quality of life and functional capacity after acute myocardial infarction: a randomized controlled trial. Can J Cardiol 2015;31:308-13. 
47 Santaularia N, Caminal J, Arnau A, et al. The efficacy of a supervised exercise training programme on readmission rates in patients with myocardial ischemia: results from a randomised controlled trial. Eur $J$ Cardiovasc Nurs 2017;16:201-12.

48 Hassan A, Nahas N. Efficacy of cardiac rehabilitation after percutaneous coronary intervention. Int J Pharmtech Res 2016:9:134-41.

49 Salavati M, Fallahinia G, Vardanjani AE, et al. Comparison between effects of home based cardiac rehabilitation programs versus usual care on the patients' health related quality of life after coronary artery bypass graft. Glob J Health Sci 2015;8:196-202.

50 Vieira Ágata, Melo C, Machado J, et al. Virtual reality exercise on a home-based phase III cardiac rehabilitation program, effect on executive function, quality of life and depression, anxiety and stress: a randomized controlled trial. Disabil Rehabil Assist Technol 2018;13:112-23.

51 Duan YP, Liang W, Guo L, et al. Evaluation of a web-based intervention for multiple health behavior changes in patients with coronary heart disease in home-based rehabilitation: pilot randomized controlled trial. J Med Internet Res 2018;20:e12052.

52 Wong EM, Chair SY, Leung DY, et al. Home-based interactive ehealth educational intervention for middle-aged adults to improve total exercise, adherence rate, exercise efficacy, and outcome: a randomised controlled trial. Hong Kong Med J 2018;24:34-38.

53 Seki E, Watanabe Y, Sunayama S, et al. Effects of phase III cardiac rehabilitation programs on health-related quality of life in elderly patients with coronary artery disease: juntendo cardiac rehabilitation program (J-CARP). Circ J 2003;67:73-7.

54 SF-36.org. FAQ: is there one summary score that is a combined score for the various subscales in the SF- $36 \AA$ so that a single score could be used for each patient? Available: http://www.webcitation. org/6cfeefPkf [Accessed 01 Oct 2019].
55 Lins L, Carvalho FM. Sf-36 total score as a single measure of health-related quality of life: scoping review. SAGE Open Med 2016;4:205031211667172.

56 Abell B, Zecchin R, Gallagher R. Making sense of the unfavourable systematic review of exercise-based cardiac rehabilitation in the modern era: how should we proceed? Heart Lung Circ 2019;28:204-6.

57 Shrier I, Steele RJ, Verhagen E, et al. Beyond intention to treat: what is the right question? Clin Trials 2014;11:28-37.

58 Stewart AL, Greenfield S, Hays RD, et al. Functional status and wellbeing of patients with chronic conditions. results from the medical outcomes study. JAMA 1989;262:907-13.

59 Hays RD, Morales LS. The RAND-36 measure of health-related quality of life. Ann Med 2001;33:350-7.

60 Wyrwich KW, Bullinger M, Aaronson N, et al. Estimating clinically significant differences in quality of life outcomes. Qual Life Res 2005;14:285-95.

61 Johnston BC, Patrick DL, Thorlund K, et al. Patient-reported outcomes in meta-analyses-part 2: methods for improving interpretability for decision-makers. Health Qual Life Outcomes 2013;11:211.

62 Wyrwich KW, Spertus JA, Kroenke K, et al. Clinically important differences in health status for patients with heart disease: an expert consensus panel report. Am Heart J 2004;147:615-22.

63 Dixon T, Lim LLY, Oldridge NB. The MacNew heart disease healthrelated quality of life instrument: reference data for users. Qual Life Res 2002;11:173-83.

64 Benzer W, Höfer S, Oldridge NB. Health-related quality of life in patients with coronary artery disease after different treatments for angina in routine clinical practice. Herz 2003;28:421-8.

65 Oldridge N, Perkins A, Marchionni N, et al. Number needed to treat in cardiac rehabilitation. J Cardiopulm Rehabil 2002;22:22-30. 